Available online at http://jddtonline.info

RESEARCH ARTICLE

\title{
METHYLATION PROFILING OF TUMOR SUPPRESSOR GENES INVOLVED IN LUNG CANCER
}

\author{
*Krishna Chaitanya Amajala, Vishwanath A, Kaladhar DSVGK and Bhaskar Reddy I \\ Department of Bioinformatics \& Biochemistry, GITAM Institute of Science, GITAM University, \\ Visakhapatnam-530045, Andhra Pradesh, India \\ *Corresponding author's Mail: chy2ak@gmail.com
}

Received 27 May 2012; Review Completed 03 July 2012; Accepted 11 July 2012, Available online 15 July 2012

\begin{abstract}
ABS TRACT
The causes of cancer are due to mutations of key proteins involved in cell cycle regulation, DNA repair enzymes and in some cases inactivation of tumor suppressor genes leads to the growth of tumors. The expression rates of TSGs vary in different stages of cancer as well in various cases of cancers. The inhibitions of TSGs are due to methylation of the DNA. In our present study, we found the genes which are methylated in different types of lung cancers and identified the methylation frequencies. Using the pubmeth database, we have identified the total number of genes undergoing methylation in the various lung cancers. From the total number of genes, we have identified the tumor suppressor genes which are in the methylated state leading to the inactivation of expression and promoting the tumor growth. The further focus on tumor suppressor genes which are methylated is necessary to find a novel way to activate them in the cancerous stage.

Keywords: DNA Methy lation, Tumor Suppressor Genes, Epigenetics, Lung Cancer, Gene Expression
\end{abstract}

\section{INTRODUCTION}

Cancer is a term used for diseases in which abnormal cells divide without control and are able to invade other tissues. Cancer cells can spread to other parts of the body through the blood and lymph systems ${ }^{1}$. The two main types of genes that are recognized as playing a role in cancer are oncogenes and tumor suppressor genes. Aberrant methylation is a widespread phenomenon in cancer and may be among the earliest changes to occur during oncogenesis. One of the major roles of DNA methylation in mammals is thought to be in control of gene regulation. This is because methylation within gene regulatory regions such as promoters and enhancers generally suppresses their function ${ }^{2}$. Several lines of evidence suggest that the methyl group in some cases directly interferes with the binding of transcription factors that activate transcription. In an alternative process, $\mathrm{CpG}$-binding domain (MBD) proteins bind to the methyl group and subsequently to chromatin remodeling proteins (such as histone deacetylases) to silence the gene by making it transcriptionally unavailable. Genes that are methylated tend to be packaged more tightly, which causes them to be silenced. Genes that are not methylated tend to exhibit looser packaging, which allows their expression ${ }^{3}$. Mutations in certain tumour suppressor genes are most often thought of in association with their inactivation during cancer initiation or progression; epigenetic alterations such as DNA methylation appear to be tightly linked to the sequential non-reversible events of normal tissue differentiation and organogenes is ${ }^{4}$.

Hence our objectives of the present study are to understand the lung cancer methylome which leads to identification of genes involved in causing lung cancers. Among the genes identified, tumor suppressor genes involved in lung cancer and their methylation frequencies are keyed out in different types of lung cancers.

\section{MATERIALS AND METHODS}

\subsection{Retrieval of Lung Cancer Genes Methylation Frequencies Information using PubMeth}

Pubmeth is an annotated and reviewed database of methylation in cancer. PubMeth can be queried using the web-interface http://matrix.ugent.be/pubmeth/search.html in two ways, depending on the researcher's focus: Gene-related: in which cancer types (and subtypes) the genes of interest are reported to be methylated and Cancer-related: which genes are reported to be methylated in the cancer types/subtypes. The Cancer-centric query approach is opted for the retrieval of methylated genes involving in Lung cancer. This type of search is meant to get a quick overview of the genes that are reported in the methylation context in the cancer (sub) types of interest and in which frequency, to explore methylation in the cancer types of interes $\mathrm{t}^{5}$.

\subsection{KEGG / Kyoto Encyclopedia of Genes \& Genomes Pathway Maps}

The KEGG Pathway database is a collection of graphical diagrams (KEGG pathway maps) and associated text information (KEGG pathway entries) for metabolism, various other cellular processes, and human diseases ${ }^{6}$. The KEGG pathway map is a moleculalr interaction/reaction network diagram represented in terms of the KEGG Orthology (KO) groups, so that experimental evidence in specific organisms can be generalized to other organisms through genomic information. Each map is manually drawn with in-house software called KegSketch, which generates the KGML+ file ${ }^{7}$. 


\section{Table 1: Tumor Suppressor Genes identified in Lung Cancer}

\begin{tabular}{|c|c|c|}
\hline S.no & Gene & Description \\
\hline "'i"' & $C D K N 2 A$ & Cyclin-dependent kinase inhibitor 2A (melanoma, p16, inhibits CDK4) \\
\hline 2 & RASSFI & Ras association (RalGDS/AF-6) domain family member 1 \\
\hline 3 & DAPK1 & Death-associated protein kinase 1 \\
\hline 4 & $\mathbf{R U N X 3}$ & Runt-related transcription factor 3 \\
\hline 5 & $A P C$ & Adenomatous polyposis coli \\
\hline 6 & $C A D M I$ & Cell adhesion molecule 1 \\
\hline 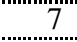 & $C D K N 2 B$ & Cyclin-dependent kinase inhibitor $2 \mathrm{~B}$ (p15, inhibits CDK4) \\
\hline 8 & $D L C 1$ & Deleted in liver cancer 1 \\
\hline 9 & $F H I T$ & Fragile histidine triad gene \\
\hline 10 & PRKCDBP & Protein kinase $C$, delta binding protein \\
\hline
\end{tabular}

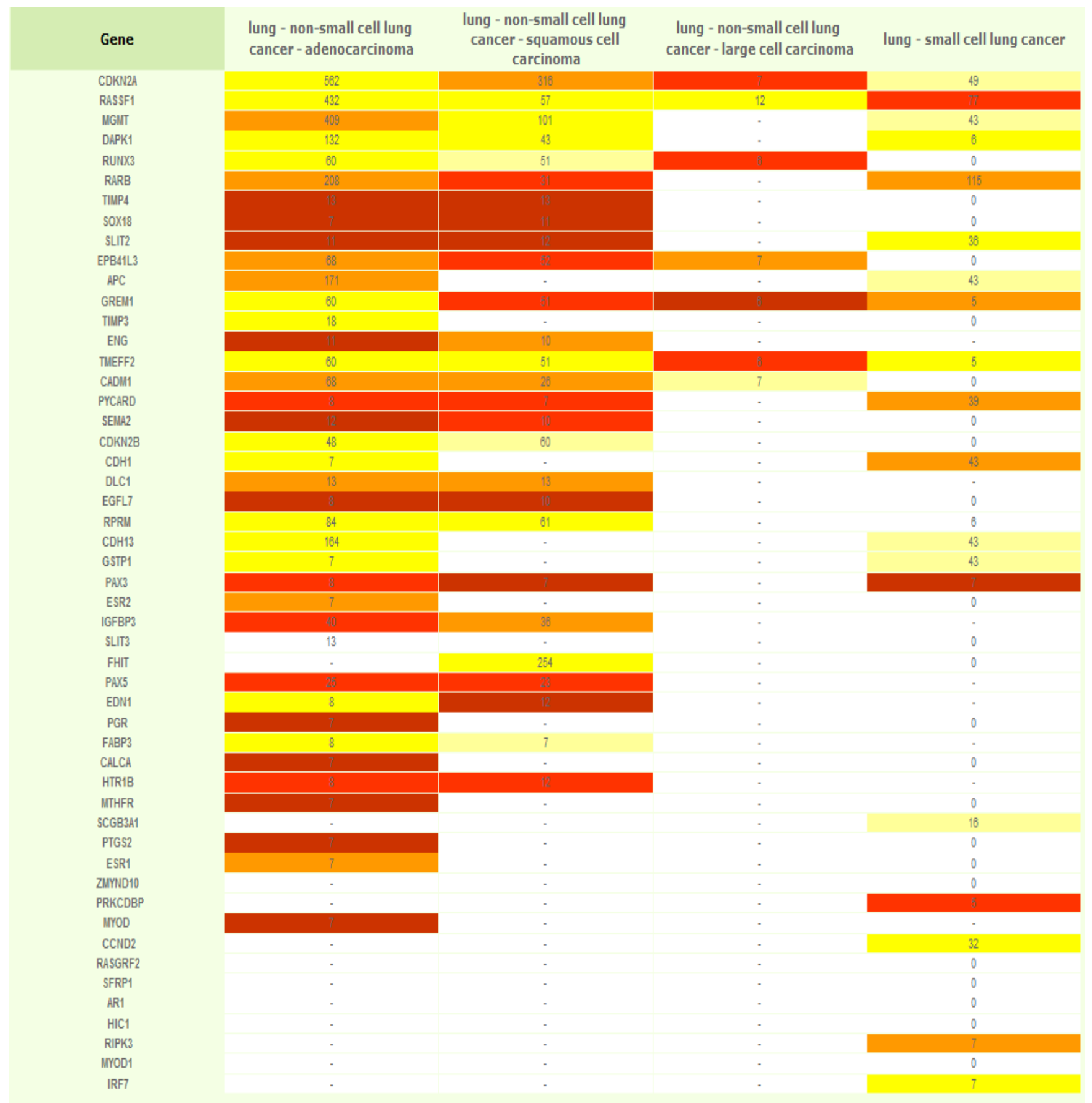




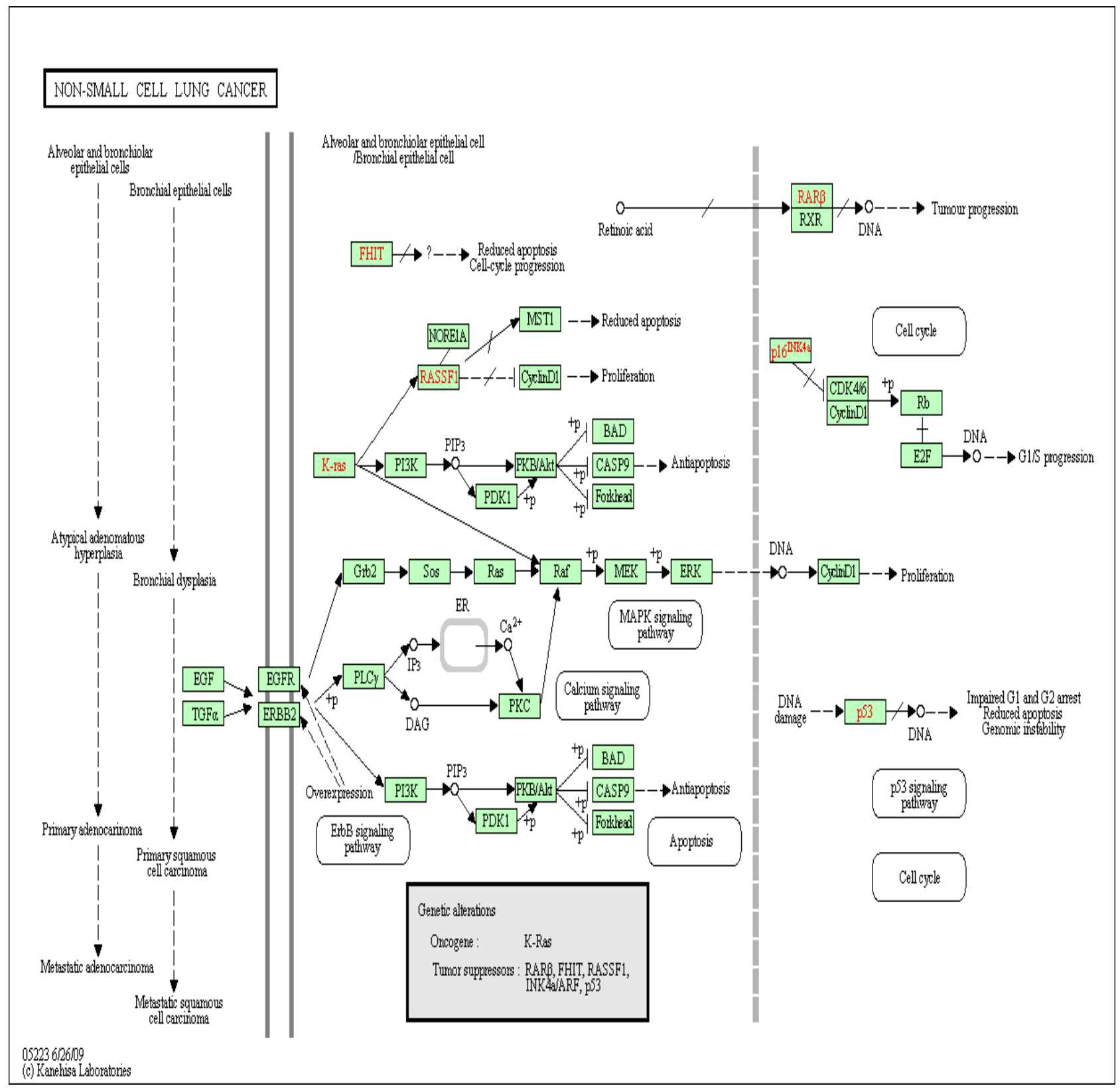

Figure 2: Non-Small cell lung cancer

Table 2: Methylation Frequencies of Tumor Suppressor Genes invol ved in Lung Cancer

\begin{tabular}{|c|c|c|c|c|}
\hline Methylation Frequencies & NSCLC - Adenocarcinoma & NS CLC - Squamous & $\begin{array}{l}\text { NSCLC - Large cell } \\
\text { carcinoma }\end{array}$ & SCLC \\
\hline $0-20 \%$ & - & $\begin{array}{l}\text { CDKN2B" } \\
\text { RUNX3 }\end{array}$ & CADM 1 & $\begin{array}{l}\text { CDKN2A } \\
\text { APC }\end{array}$ \\
\hline $20-40 \%$ & $\begin{array}{l}\text { CDKN2A; } 2 \mathrm{~B} \\
\text { RASSF1 } \\
\text { DAPK1 } \\
\text { RUNX3 }\end{array}$ & $\begin{array}{l}\text { RASSF1 } \\
\text { DAPK1 } \\
\text { FHIT }\end{array}$ & RASSF1 & DAPK1 \\
\hline $40-60 \%$ & $\begin{array}{c}\text { APC } \\
\text { CADM } 1 \\
\text { DLC } 1\end{array}$ & $\begin{array}{l}\text { CDKN2A" } \\
\text { CADM1 } \\
\text { DLC1 }\end{array}$ & - & - \\
\hline $60-80 \%$ & - & - & $\begin{array}{l}\text { CDKN2A } \\
\text { RASSF1 } \\
\text { RUNX3 }\end{array}$ & $\begin{array}{c}\text { RASSF1 } \\
\text { PRKCDBP }\end{array}$ \\
\hline $80-100 \%$ & - & - & - & - \\
\hline
\end{tabular}




\section{SMALL CELL LUNG CANCER}

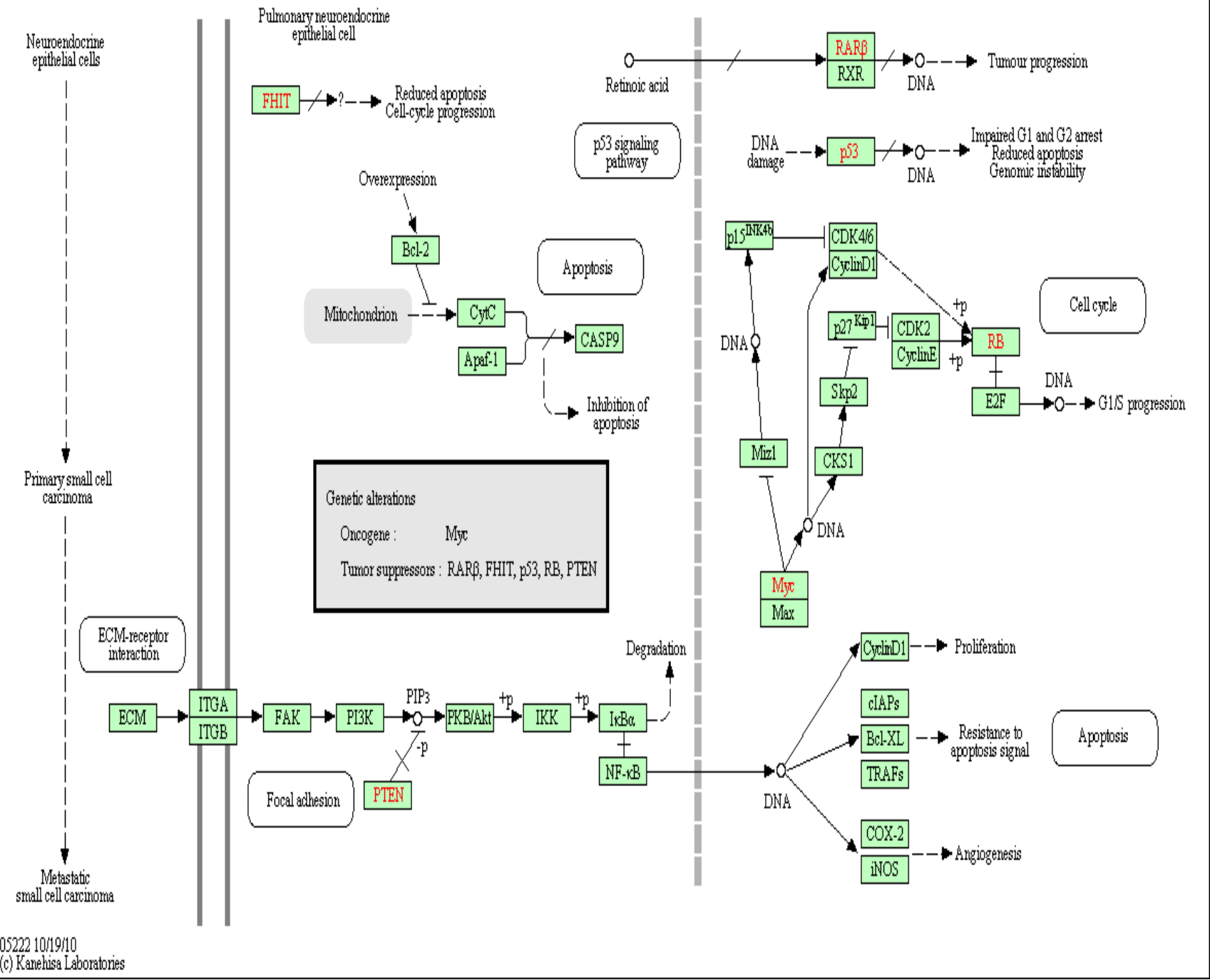

Figure 3: Small cell lung cancer

\section{DISCUSS ION}

\subsection{Identifying the Tumor Suppressor Genes and their Methylation Frequencies}

By using PubMeth database, the genes which are found to be methylated in various lung cancer cell lines are depicted in the figure 1. There are two different types of lung cancers i.e.., are NSCLC - Non-Small Cell Lung Cancer \& SCLC - Small Cell Lung Cancer. The subtypes of NSCLC are: NSCLC-Adenocarcinoma, NSCLC-Squamous, and NSCLC-Large Cell Carcinoma. Among those identified genes, the TSGs are recognized to know how many TSGs are involved in various types of lung cancers. Specific TSGs show different methylation status in respective types of lung cancers. Such kinds of methylation frequencies are also observed in other cancer types where the same TSGs may possess other methylation status.

\subsection{Methylation Frequencies of TS Gs in Lung Cancer}

In different types of lung cancers, it has been observed that no TSGs are likely having the same methylation frequencies / status. In each type of lung cancer different TSGs have different methylation status and these methylation statuses differ for various kinds of cancers also. The identified TSGs are tabulated in the table $\mathbf{1}$ and their respective methylation frequencies are tabulated in table 2. The dark red coloration indicates the highest methylation frequency of the genes. Among the identified TSGs, the RASSF1 \& CDKN2A TSGs are highly methylated and involved in both Non-Small Cell \& Small Cell Lung Cancers.

\subsection{KEGG - NSCLC \& S CLC Pathway Maps}

The identification of TSGs in the cancer pathway maps gives an insight to the connectivity of other genes as well associative cellular mechanisms can be understood well. By the pathways, the ability to find the connectivity to other cellular pathways such as apoptosis, cell-cycle progression, etc can be graphically depicted. The pathway 
maps provide focused information about the various genes associated, colligated and responsible for various cellu lar states. From the NSCLC pathway map (figure 2: NSCLCNon-S mall Cell Lung Cancer Pathway), it can be depicted that the genes FHIT \& RASSF1 are indirectly involved in controlling the various functions like reduced apoptosis, cell-cycle progression \& proliferation through other key proteins like CyclinD1 \& MST1. From the methylation frequencies analyses, the both TSGs are highly methylated which results them in uncontrolling the key functions of cell-cycle progression \& proliferation. In the same manner, the SCLC pathway map (figure 3: SCLC-S mall Cell Lung Cancer Pathway), also provides information regarding the other TSGs like Myc, RB, p53, PTEN along with the FHIT \& CDKN2A genes which are methylated in both the SCLC \& NSCLC. These TSGs play a critical functional role in inhibition of apoptosis, degradation, tumor progression, proliferation and angiogenes is.

\section{REFERENCES}

1. Amajala Krishna Chaitanya, MTP. Mahalakshmi, I. Bhaskar Reddy, Kaladhar DSVGK, Methylation profiling of tumor suppressor genes involved in various cancers and structural analy sis of RASSF1 protein, IJCBPR, 2012, 2(1), 208-212.

2. Susan J Clark and John Melki, DNA methylation and gene silencing in cancer: which is the guilty party?, Oncogene, 2002, 21, 35, Pages 5380-5387.

3. Bird A, Wolffe AP. Methylation-induced Repression: Belts, Braces and Chromatin. Cell. 1999. 99: 451-454.

4. Garinis GA, Patrinos GP, Spanakis NE, Menounos PG, DNA hypermethylation: when tumour suppressor genes go silent. Hum Genet. 2002, Aug;111(2):115-27.

5. Mate O, Leander VN, Tim DM, Gerben M, Sofie B and Wim VC, PubMeth: a cancer methylation database

\section{CONCLUS ION}

By the PubMeth database studies, we can conclude that the minimum numbers of genes in lung cancer cell lines involved in methylation are 44 genes. Among these 44 genes, 10 are identified as Tumor Suppressor Genes, and their detailed annotated information has been obtained through Genecards database. The 10 specific TSGs having different methylation frequencies have been observed. This indicates that methylation states are varied from one cancer to another type of cancer. The pathway maps focus the colligation of other genes and exploring differential gene connectivity and pathway conservation in identifying the novel ways to activate the tumor suppres sor genes.

\section{ACKNOWLEGEMENTS}

We would like to thank GITAM University for providing Bioinformatics lab facility to carry out the research work.

\section{CONFLICT OF INTER ES T - None}

combining text-mining and expert annotation, Nucleic Acids Research, 2008, Vol. 36, D842-D846.

6. Kanehisa M, Goto S, KEGG: Ky oto Ency clopedia of Genes and Genomes. Nucleic Acids Res., 2000, 28, 27-30.

7. Kanehisa M, Goto S, Furumichi M, Tanabe M, Hirakawa M KEGG for representation and analysis of molecular networks involving diseases and drugs. Nucleic Acids Res., 2010, 38, D355-D360.

8. Carlo S, Brueckner B, Tanja M, Helga S, Frank L, Functional Diversity of DNA Methyltransferase Inhibitors in Human Cancer Cell Lines, Cancer Res; 2006, 66: (5). March 1, 2794-2800.

9. Ivy FL. Tsui Raj Chari, Timon PH. Buys Wan L. Lam, Public Databases and Software for the Pathway Analysis of Cancer Genomes. Cancer Informatics, 2007, 3, 379-397. 